\title{
Propriedades Mecânicas de Tração de Compósitos Poliéster/Tecidos Híbridos Sisal/Vidro
}

\author{
Laura H. de Carvalho \\ Departamento de Engenharia de Materiais, UFCG \\ Wilma S. Cavalcanti \\ CDEP, UFPB
}

\begin{abstract}
Resumo: O desempenho e o custo de compósitos podem ser alterados por hibridização e, neste sentido, é relativamente comum o uso combinado de fibras e reforços minerais no desenvolvimento destes materiais. No presente trabalho o desempenho mecânico de compósitos poliéster insaturado/ tecidos híbridos sisal-vidro foram investigados em função do teor de fibra e direção do teste. Foram confeccionados três tecidos híbridos (com 30, 40 e $50 \%$ em peso de vidro) com fios de sisal no urdume e fibras de vidro na trama. Os compósitos foram moldados por compressão à temperatura ambiente com os tecidos alinhados. Os resultados indicam que houve um aumento nas propriedades mecânicas de tração de todos os compósitos com o aumento do teor de fibras. Para os compósitos reforçados por tecidos com baixo teor de fibra de vidro, as propriedades tenderam a ser mais elevadas quando os testes foram conduzidos na direção do sisal, enquanto que para os tecidos com elevado teor de vidro, o oposto foi observado. Estes comportamentos foram associados ao teor de fibra de vidro na direção do teste e ao diâmetro das fibras de sisal. Em baixos teores de fibra o sisal agiria como inclusão ou defeito, prejudicando as propriedades mecânicas; em elevados teores as propriedades do vidro suplantariam os defeitos provocados pelo sisal.
\end{abstract}

Palavras-chave: Tecido hibrido, compósito, fibra vegetal, poliéster, propriedades tênseis.

\section{Properties of Polyester/Hibrid Sisal-Glass Fabrics}

Abstract: Hybridization can alter both mechanical performance and cost of polymer composites, and novel composite materials can be obtained by the combination of both fibrous and mineral reinforcements. In the present work the mechanical performance of unsaturated polyester/hybrid sisal-glass fabrics was determined as a function of fibre content and test direction. Three different hybrid fabrics $(30,40$ and $50 \% \mathrm{w} / \mathrm{w}$ glass content) with sisal strings in the warp and glass roving in the weft were hand weaved. Aligned fabric compression moulded composites were obtained at room temperature. The results showed enhanced properties with fibre content for all composites under investigation. Composites reinforced by fabrics with low glass fibre content exhibited lower properties when tested along the glass fibre direction than when tested along the sisal fibre direction and, at high glass fibre content the opposite trend was observed. These behaviours were associated with the low glass fibre content along the test direction and to the high sisal fibre diameter. At low glass fibre content the sisal fibres would act as inclusions or defects, thereby lowering the mechanical performance of the composite; at high glass fibre contents the superior mechanical properties of the glass would surpass the defects caused by sisal fibres.

Keywords: Hybrid fabrics, composite, vegetable fibre, polyester, mechanical properties.

\section{Introdução}

O uso de fibras vegetais como reforço em compósitos poliméricos com o objetivo de substituir total ou parcialmente as fibras sintéticas tem recebido muita atenção nos últimos anos ${ }^{[1-7]}$. Isto porque as fibras vegetais apresentam vantagens importantes como: baixo custo, baixa densidade, boa resistência mecânica, baixa abrasividade aos equipamentos de processo e também por serem provenientes de fontes renováveis de matéria-prima, disponíveis em todo o mundo. Além disto, as fibras vegetais são biodegradáveis e não são tóxicas ou poluentes, de modo que o seu descarte não acarreta problemas ambientais. As principais desvantagens no emprego dessas fibras em compósitos poliméricos são relacionadas à natureza polar e hidrofílica bem como à susceptibilidade destas fibras a ataques de fungos e bactérias. A natureza hidrofílica das fibras vegetais resulta em pobre umectação e adsorção de grande parte dos polímeros em sua superfície, resultando em fraca adesão interfacial polímero-fibra $^{[1-7]}$.

A qualidade da interface fibra-matriz é significativa para a aplicação de fibras vegetais como reforço para plásticos.

Autor para correspondência: Laura H. de Carvalho, Departamento de Engenharia de Materiais, UFCG, Caixa Postal 10034, CEP: 58109-970, Campina Grande, PB. E-mail: laura@dema.ufcg.edu.br 
Métodos físicos e químicos podem ser usados, como promotores de adesão entre a matriz e a fibra, para melhorar a interface com diferentes eficiências ${ }^{[8-10]}$. Uma outra maneira de se obter produtos com as propriedades desejadas é a hibridização de reforços, seja através da combinação de laminados ou da mistura de fibras ${ }^{[11-14]}$. Compósitos laminados híbridos com fibras vegetais e fibras de vidro são bastante resistentes e mais leves do que os reforçados unicamente por fibras de vidro. Este tipo de compósito, por outro lado, tende a apresentar problemas de delaminação. Uma forma de não localizar este problema é o uso de misturas de fibras e da confecção de tecidos híbridos. Tecidos híbridos de elevado desempenho a base de fibras de carbono, vidro e kevlar têm sido bastante utilizados, principalmente na área aeronáutica, como reforço em polímeros ${ }^{[13-16]}$. Raras são as referências sobre o uso de tecidos híbridos a base de fibras vegetais $^{[11-13,18-19]}$. Nosso grupo ${ }^{[6,7,17-19]}$ obteve e reportou valores $30-40 \%$ superiores aos demandados pelas montadoras para a resistência de compósitos de matriz poliéster a serem utilizados em carcaças de caminhões. Estes resultados foram obtidos tanto para compósitos reforçados por tecidos híbridos à base de fibras vegetal/vegetal, quanto para os vegetal/ sintética.

Neste trabalho, foram manufaturados compósitos poliméricos, originais, moldados por compressão, reforçados por tecidos híbridos sisal e vidro, dispostos em camadas alinhadas. O desempenho mecânico destes compósitos foi avaliado em função do teor de reforços e da direção do teste, de modo que tanto o efeito do teor total de fibras quanto do teor relativo e identidade das fibras alinhadas na direção do teste puderam ser investigados.

\section{Experimental}

Utilizou-se como matriz uma resina poliéster insaturada (Resapol 10-116), do tipo ortoftálica e pré-acelerada, dissolvida em monômero de estireno, fornecida pela RESANA S.A., curada com peróxido de metil etil cetona (MEK-P) em concentração de $1 \%$ em peso.

Esta resina, segundo o fabricante, é do tipo insaturada, ortoftálica, rígida, totalmente polimerizável, apresentando baixa reatividade, baixa viscosidade e pré-acelerada. É uma resina de uso geral, indicada para aplicações em piscinas, banheiras, peças automotivas, náuticas e acessórios reforçados com fibras de vidro, moldados por laminação manual ou a pistola.

Os reforços consistiam de tecidos híbridos sisal/vidro tramados em tear manual no Lar do Garoto, reformatório juvenil sediado no município de Lagoa Seca, PB. Fios de sisal $(700 \mathrm{~m} / \mathrm{Kg})$ fornecidos pela BRASCORDA S.A foram dispostos no urdume e mechas (roving) de fibras de vidro (111A 408X1) fornecidas pela OWENS CORNING do BRASIL, foram empregadas na trama. Segundo o fornecedor, o 111A é um roving direto, especialmente projetado para os processos de enrolamento (filamento winding), tecelagem, reforços de cabos telefônicos e pultrusão, sendo compatível com resinas poliéster, viniléster e epóxi. As fibras são tratadas superficialmente para rápida molhagem e facilidade de processamento. Tratamento químico, de alta performance, é aplicado para otimizar a adesão do vidro na matriz polimérica, para se adequar às necessidades de custo e desempenho. $\mathrm{O}$ roving de fibra de vidro 111A408 apresenta $408 \mathrm{Texg} / \mathrm{Km}$, $16 \mu \mathrm{m}$ de diâmetro e 800 filamentos por cabo ${ }^{[20]}$.

Foram confeccionados três tecidos possuindo distintas quantidades relativas sisal/vidro. $\mathrm{O}$ tear foi operado utilizando-se um pente \#18 e um fio de sisal por pua. O teor relativo de fibras foi modificado variando-se o número de mechas de fibras de vidro por passagem na trama, de modo que tecidos com teores ponderais relativos sisal/vidro de $68 \% / 32 \%$; $58 \% / 42 \%$ e $51 \% / 49 \%$, respectivamente, para tecidos contendo uma, duas ou três mechas de fibra de vidro por passagem na trama, foram obtidos. Amostras $10 \times 10 \mathrm{~cm}$ dos tecidos híbrido sisal/vidro apresentavam: a) 38 cordões de sisal e 22 mechas de vidro para o tecido com 1 mecha de vidro por passagem na trama; b) 36 cordões de sisal e 20 mechas (dupla) de vidro e c) 36 cordões de sisal e 19 mechas (tripla) de vidro.

Compósitos com teores, totais e relativos, distintos de fibra foram preparados por moldagem por compressão. Os tecidos híbridos sisal/vidro foram cortados nas dimensões internas do molde ( $150 \times 180 \mathrm{~mm})$ e pesados. A superfície do molde foi untada com uma fina camada de cera de carnaúba para atuar como desmoldante. Parte da resina, previamente misturada com o catalisador, foi vertida no molde; o tecido híbrido selecionado foi disposto sobre a resina, impregnado com o auxílio de uma espátula e por fim foi colocado o restante da resina. Quando a resina estava próxima do ponto de gelificação o molde foi fechado e o conjunto prensado em prensa hidráulica uniaxial sob força de 8 toneladas à temperatura ambiente durante 4 horas. O compósito foi desmoldado e corpos-de-prova foram usinados (serrados com serra manual) a partir das placas obtidas 48 horas após a desmoldagem. Compósitos laminados (150 x 180 × $3 \mathrm{~mm}$ ) com até 3 camadas de tecidos foram confeccionados utilizando-se o mesmo procedimento, tendo-se o cuidado de manter os tecidos com as fibras de mesma identidade (sisal ou vidro) numa única direção e impregnando-se completamente cada camada individual para evitar a formação de bolsões de resina ou de ar.

Os resultados reportados para os ensaios mecânicos em tração são valores médios de no mínimo cinco corpos-deprova $(110 \times 12,7 \times 3 \mathrm{~mm})$ testados segundo a norma ASTM 3039 à temperatura ambiente, em máquina universal de ensaios Lloyd LR /10KN operando com velocidade de deslocamento de travessa de $1 \mathrm{~mm} / \mathrm{min}$.

\section{Resultados e Discussão}

Os resultados obtidos estão mostrados na Tabela 1 e ilustrados nas Figuras 1-3.

Fica evidenciado que a resistência mecânica dos compósitos investigados aumenta com o teor total de fibras e que, de modo geral, propriedades superiores foram obtidas 
para as amostras com elevados teores de fibras de vidro testadas na direção do vidro. Este tipo de comportamento foi verificado anteriormente para compósitos afins, reforçados por tecidos híbridos a base de algodão/juta e juta/vidro ${ }^{[6,22]}$.

A resistência à tração de materiais compósitos reforçados por fibras depende, principalmente, da resistência e módulo das fibras de reforço; teor, orientação e comprimento das fibras; estabilidade química e resistência da matriz e da interface fibra/matriz. Em tecidos tramados e urdidos, o grau de entrelaçamento das fibras no tecido é determinado pelo padrão da tecedura, e as fibras deformam ou dobram de maneira regular e reversível.

O motivo para o elevado desempenho mecânico exibido pelos compósitos testados na direção longitudinal às fibras na trama (vidro) é atribuído ao fato que, neste arranjo, as fibras não só encontram-se perfeitamente orientadas e alinhadas, como também, suas propriedades são superiores às das fibras dispostas no urdume (sisal). Além disto, as fibras de vidro já são superficialmente modificadas de modo que sua adesão com a matriz poliéster é superior à exibida pelas fibras de sisal. Este conjunto de fatores - propriedades individuais, maior alinhamento e melhor adesão - facilitam a transferência efetiva de tensões da matriz para as fibras. Quando os laminados foram testados paralelamente às fibras da urdidura (sisal) observou-se que a resistência das fibras nesta direção tendeu a ser inferior à oferecida pelas fibras da trama, o que era esperado.

As Figuras 1a e 1b ilustram o comportamento da resistência à tração dos compósitos investigados em função do teor de fibra e direção do teste, isto é, compósitos testados na direção das fibras de sisal (a) e de vidro (b).

Os resultados indicam que, para cada tecido utilizado como reforço, isto é, tecidos com 1, 2 ou 3 mechas de vidro na trama, houve um aumento na resistência dos compósitos com o aumento no teor de fibras e que, para um mesmo teor relativo de fibras na direção do teste, propriedades mais elevadas foram obtidas quando os ensaios realizados na direção das fibras de vidro (Figura 1b).

Tabela 1. Propriedades Mecânicas de Compósitos Poliéster/Tecido Híbrido Sisal-Vidro

\begin{tabular}{|c|c|c|c|c|c|c|c|}
\hline Tipo de Tecido & $\begin{array}{c}\text { Número de } \\
\text { Camadas } \\
\#\end{array}$ & $\begin{array}{c}\text { Teor Total } \\
\text { de Fibra } \\
(\%)\end{array}$ & $\begin{array}{l}\text { Direção } \\
\text { do Teste }\end{array}$ & $\begin{array}{c}\text { Teor de Fibra } \\
\text { na Direção } \\
\text { do Teste } \\
(\%)\end{array}$ & $\begin{array}{c}\text { Resistência à } \\
\text { Tração } \\
\text { (MPa) }\end{array}$ & $\begin{array}{c}\text { Módulo de } \\
\text { elasticidade } \\
\text { (GPa) }\end{array}$ & $\begin{array}{c}\text { Alongamento } \\
\text { na Ruptura } \\
(\%)\end{array}$ \\
\hline Matriz & 0 & 0 & - & - & $32,8 \quad 2,1$ & $1,28 \quad 0,16$ & $2,8 \quad 0,2$ \\
\hline \multirow{6}{*}{$\begin{array}{l}\text { Sisal/1mecha } \\
\text { de Vidro }\end{array}$} & 1 & 20 & Sisal & 13,6 & $25,4 \pm 2,4$ & $2,44 \pm 0,30$ & $6,4 \pm 1,5$ \\
\hline & 1 & 20 & Vidro & 6,4 & $19,0 \pm 4,2$ & $1,50 \pm 0,31$ & $10,4 \pm 2,2$ \\
\hline & 2 & 33 & Sisal & 22,4 & $42,9 \pm 4,1$ & $2,77 \pm 0,17$ & $6,7 \pm 0,8$ \\
\hline & 2 & 33 & Vidro & 10,6 & $21,2 \pm 5,4$ & $1,39 \pm 0,16$ & $9,4 \pm 2,5$ \\
\hline & 3 & 45 & Sisal & 30,6 & $45,2 \pm 5,9$ & $2,54 \pm 0,21$ & $6,4 \pm 0,6$ \\
\hline & 3 & 45 & Vidro & 14,4 & $40,5 \pm 4,3$ & $0,95 \pm 0,41$ & $14,2 \pm 0,5$ \\
\hline \multirow{6}{*}{$\begin{array}{l}\text { Sisal/2mechas } \\
\text { de Vidro }\end{array}$} & 1 & 28 & Sisal & 16,0 & $42,8 \pm 3,6$ & $2,93 \pm 0,21$ & $6,1 \pm 0,6$ \\
\hline & 1 & 28 & Vidro & 12,0 & $35,6 \pm 9,7$ & $1,77 \pm 0,29$ & $9,3 \pm 2,4$ \\
\hline & 2 & 37 & Sisal & 21,5 & $45,7 \pm 4,2$ & $2,92 \pm 0,13$ & $6,1 \pm 0,7$ \\
\hline & 2 & 37 & Vidro & 15,5 & $47,2 \pm 6,9$ & $2,03 \pm 0,13$ & $9,2 \pm 2,1$ \\
\hline & 3 & 59 & Sisal & 34,0 & $64,1 \pm 2,5$ & $3,72 \pm 0,26$ & $6,1 \pm 0,2$ \\
\hline & 3 & 59 & Vidro & 25,0 & $40,1 \pm 7,2$ & $2,08 \pm 0,24$ & $8,8 \pm 2,6$ \\
\hline \multirow{6}{*}{$\begin{array}{c}\text { Sisal/3mechas } \\
\text { de Vidro }\end{array}$} & 1 & 30 & Sisal & 15,3 & $24,8 \pm 4,4$ & $2,79 \pm 0,16$ & $3,6 \pm 0,4$ \\
\hline & 1 & 30 & Vidro & 14,7 & $63,5 \pm 4,8$ & $2,08 \pm 0,14$ & $8,9 \pm 1,2$ \\
\hline & 2 & 40 & Sisal & 20,4 & $45,3 \pm 1,5$ & $3,02 \pm 0,10$ & $6,2 \pm 0,5$ \\
\hline & 2 & 40 & Vidro & 19,6 & $95,5 \pm 11,1$ & $2,49 \pm 0,16$ & $10,6 \pm 1,3$ \\
\hline & 3 & 57 & Sisal & 29,0 & $52,6 \pm 7,4$ & $3,72 \pm 0,33$ & $5,7 \pm 0,9$ \\
\hline & 3 & 57 & Vidro & 28,0 & $123,8 \pm 13,7$ & $3,83 \quad 0,34$ & $9,9 \pm 0,5$ \\
\hline
\end{tabular}


Resultados aparentemente anômalos foram obtidos para compósitos reforçados pelos tecidos com uma (32\% vidro/ $68 \%$ sisal) e duas mechas ( $42 \%$ vidro $/ 58 \%$ sisal) de fibra de vidro na trama. Nestes casos, surpreendentemente, as propriedades $(\sigma$ e E) dos compósitos testados na direção do sisal (Figura 1a) foram superiores às do mesmo material testado na direção do vidro (Figura 2b). Além disto, para o tecido manufaturado com uma mecha de fibra de vidro, em baixo teor total de fibras (20\% em massa, ou seja, $13,6 \%$ de sisal e $6,5 \%$ de vidro) resistências inferiores às da matriz foram obtidas. Estes dados se opõem aos resultados obtidos anteriormente para tecidos híbridos juta/vidro onde as propriedades dos compósitos testados na direção da fibra vegetal foram sempre inferiores às obtidas para os testados na direção do vidro ${ }^{[19]}$. Acredita-se que os motivos para esta discrepância estejam associados aos elevados diâmetros e resistência mecânica das fibras de sisal utilizadas e ao baixo teor relativo de fibras de vidro destes tecidos. As mechas (roving) de fibras de vidro utilizadas são compostas por finos filamentos, superficialmente tratados para terem boa adesão com a matriz. Por outro lado, o fio de sisal empregado na confecção dos tecidos não foi quimicamente tratado, de modo que, devido às suas características hidrofílicas, não possui boa adesão com a matriz além de ser composto por um conjunto de fibras retorcidas e de difícil impregnação. Outra possível causa para esta discrepância seria a impregnação irregular das fibras nas regiões de entrecruzamento dos fios que compõe o tecido. Nos compósitos reforçados pelo tecido com uma mecha de vidro, por exemplo, teores totais de fibra de 20 e $33 \%$ em peso implicam em teores de fibras de vidro na direção do teste de 6,4 e 10,6\% respectivamente. Abaixo de uma concentração crítica de fibras, as propriedades do compósito são dominadas pela matriz e propriedades inferiores às da matriz são obtidas e previstas ${ }^{[21-23]}$. Acredita-se que este conjunto de fatores seja responsável pelos resultados obtidos.

As Figuras 2a e 2b ilustram o efeito do tipo de tecido e do teor individual de fibras no módulo de elasticidade dos compósitos em função da direção do teste.

Os resultados são semelhantes aos obtidos para a resistência à tração e indicam que o módulo de elasticidade dos
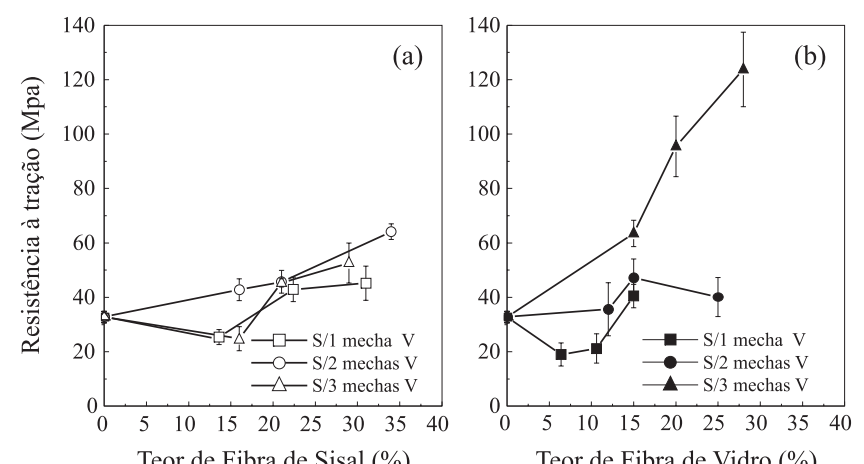

Figura 1. Resistência à Tração dos compósitos em função do teor individual de fibras e direção do teste. S/1mecha V - SL e S/1mecha V - VL, compósito sisal/1mecha de vidro testado na direção longitudinal ao sisal (a) e ao vidro (b), respectivamente. compósitos investigados tende a aumentar com o teor e identidade das fibras e que, de modo geral, o módulo é maior para os testes realizados na direção das fibras de vidro (Figura $2 b$ ). Os compósitos mais rígidos foram aqueles reforçados por tecidos híbridos com três mechas de vidro. Este comportamento era o esperado já que o módulo das fibras de vidro é superior ao das fibras de sisal ${ }^{[18,19]}$. Novamente, resultados destoantes desta tendência foram apresentados pelos compósitos reforçados com os tecidos tendo uma ou duas mechas de fibras de vidro na trama. Enquanto os compósitos reforçados pelo tecido com uma mecha de fibra de vidro na trama, testados na direção do vidro, apresentaram uma redução do módulo com o aumento do teor de fibras, os reforçados por tecidos com duas mechas de vidro aumentaram, mas em ambos os casos módulos inferiores aos obtidos para o compósito testado na direção do sisal foram obtidos. Acredita-se que este resultado inesperado, e aparentemente incongruente, esteja associado aos seguintes fatores: a) baixo teor relativo de fibras de vidro nestes tecidos; b) defeitos na orientação das fibras de vidro na região de entrecruzamento entre as fibras do urdume (sisal) e da trama (vidtro). Isto porque, tecidos de trama simples, as fibras do urdume fazem um movimento ondulatório passando por cima e por baixo das fibras da trama. Portanto, sendo o diâmetro do sisal bastante superior ao da fibra de vidro, na região de entrecruzamento o alinhamento das fibras de vidro fica bem mais comprometido do que o das fibras de sisal e; c) a algumas dificuldades na obtenção dos corposde-prova que pareciam muito mais propensos a trincas e fissuras do que os reforçados por tecidos com teor mais elevado de fibras de vidro.

As Figuras $3 \mathrm{a}$ e $3 \mathrm{~b}$ ilustram o comportamento do alongamento na ruptura dos compósitos em função do teor de fibras e da direção do teste. Os dados indicam que o alongamento tendeu a aumentar com o teor de fibras. Resultados semelhantes foram reportados em sistemas afins ${ }^{[6,17-19]}$ e foram tomados como indicativos de uma tenacificação do material pela incorporação das fibras. $\mathrm{O}$ aumento mais significativo nesta propriedade para os compósitos testados na direção das fibras de vidro (Figura 3b) é atribuído à melhor molhabilidade
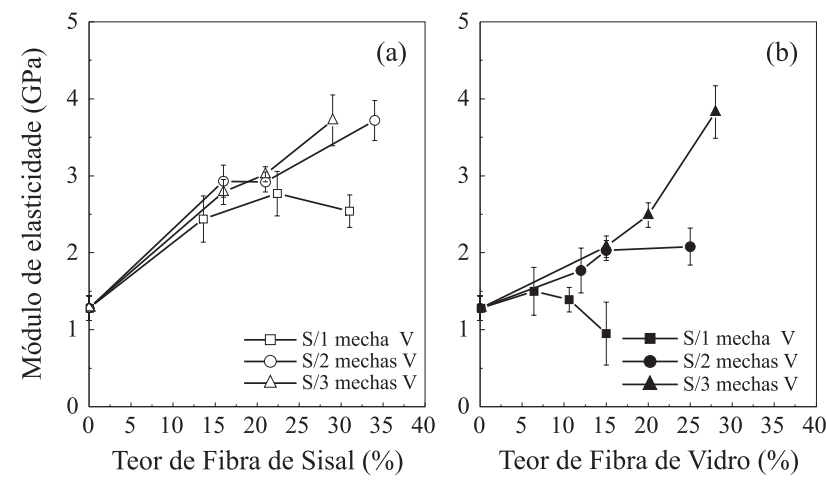

Figura 2. Módulo de elasticidade dos compósitos em função do teor individual de fibras e direção do teste. S/1mecha V - SL e S/1mecha V - VL, compósito sisal/1mecha de vidro testado na direção longitudinal ao sisal (a) e ao vidro (b), respectivamente. 

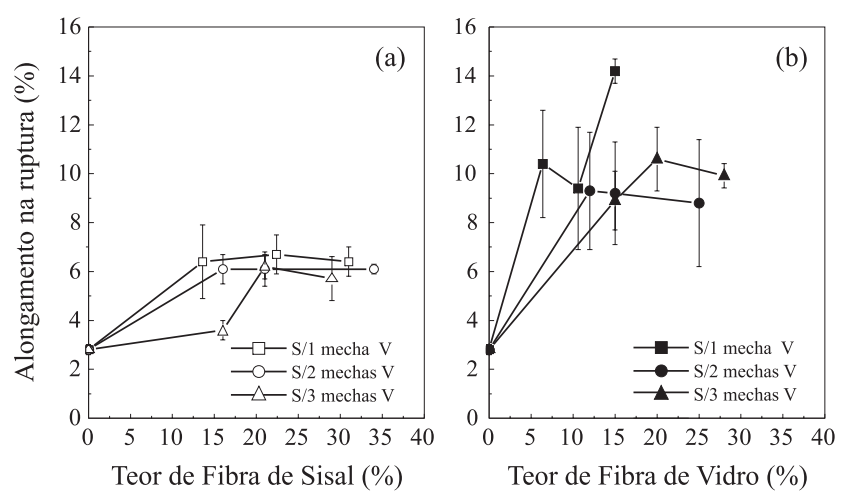

Figura 3. Alongamento na ruptura de compósitos poliéster reforçados com tecido híbrido sisal/vidro em função do teor total de fibras e direção do teste. $\mathrm{S} / 1$ mecha V - SL e S/1mecha V - VL, compósito sisal/1mecha de vidro testado na direção longitudinal ao sisal (a) e ao vidro (b), respectivamente.

das fibras de vidro pela matriz já que estas são superficialmente tratadas e as fibras de sisal não foram modificadas.

\section{Conclusões}

Foram desenvolvidos compósitos de matriz poliéster insaturado reforçados por tecidos híbridos tendo sisal no urdume e diferentes teores de fibras de vidro na trama. De modo geral, as propriedades em tração aumentaram com o teor e identidade das fibras, sendo mais elevadas para os compósitos reforçados por tecidos com elevado teor de fibras de vidro e testados na direção do vidro. Surpreendentemente, a baixos teores de fibra de vidro, as propriedades dos compósitos testados na direção do sisal foram superiores às dos testados na direção do vidro, contrariando resultados anteriores obtidos em sistemas afins, reforçados por tecidos híbridos juta-vidro. Estas discrepâncias foram atribuídas às propriedades mecânicas das fibras de sisal e características morfológicas, como elevado diâmetro quando comparadas às fibras de sisal. Os dados parecem indicar uma adesão interfacial inferior para o sistema reforçado pelo híbrido sisal-vidro se comparado àquele reforçado pelo híbrido juta-vidro.

\section{Agradecimentos}

À Resana S.A., Brascorda S.A., Owens-Corning do Brasil e VI Produtos Químicos pela doação dos insumos. Ao tecelão José Leandro Gomes da Silva pela fabricação dos tecidos. Ao CNPq/PADCT pelo suporte financeiro ao projeto (projeto \# 620343/98-2), e pelas bolsas aos autores Cavalcanti (CNPq-DCR) e Carvalho (CNPq-PQ).

\section{Referências Bibliográficas}

1. Bledzki, A. K. \& Gassan, J. - Prog. Polym. Sci., 24, 221 (1999).

2. Wambua, P.:Ivens, J. \& Verpoest, I. - Comp. Sci. Technol., 63, p.1259 (2003)

3. Li, Y.; Mai, Y-W. \& Ye,L. - Comp. Sci. Technol., 60, p.2057 (2000).
4. Rong,M.Z.; Zhang, M. Q.; Liu, Y.; Yang, G. C. \& Zeng,H.M. Comp. Sci. Technol., 61, p. 1437 (2001).

5. Frollini, E.; Razera, I.A.T. - J. Appl. Polym. Sci., 91, p. 1077 (2004).

6. Medeiros, E.S.; Agnelli, J.A.M.; Joseph, K.; Carvalho, L.H.; Mattoso, L.H.C.; - J. Appl. Polym. Sci., 90, p. 1678 (2003).

7. Joseph, P.V.; Rabello, M.S.; Mattoso, L.H.C.; Joseph, K.; Thomas, S. - Comp. Sci. Technol., 62, p.1367 (2002).

8. Bledzki, A. K.; Reihmane, S. \& Gassan, J. - J. Appl. Polym. Sci., 59, p.1329 (1996).

9. Calado, V.; Barreto, D.W.; D’Almeida, J,R,M, - Polym. And Polym. Comp., 11, p.31 (2003).

10. Boynard, C.A.; Monteiro, S.N.; D’Almeida, J.R.M. - J. Appl. Polym. Sci., 12, p. 1927 (2003).

11. Carvalho, L.H.; Joseph, K. \&.Nóbrega, M. M. S. “ Reforços Híbridos em Compósitos Poliéster/Fibras Vegetais", in: Anais do $4^{\circ}$ Simpósio Argentino de Polimeros, p. 67, Cordoba - Argentina, (1999).

12. Joseph, K. \& Carvalho, L. H. - "Propriedades de Compósitos Poliéster Insaturado/Tecido Híbrido de Juta-Algodão.”, in. Anais do $5^{\circ}$ Congresso Brasileiro de Polímeros, p. 834, Águas de Lindóia - São Paulo, (1999).

13. Clark, R. A. \&.Ansell, M. P. - J. of Mat. Sci., 21, p. 269, (1996)

14. Rezende, M.C.; Silva, R.S. - Polímeros: Ciência e Tecnologia, 13, p.79 (2003).

15. Leong, K. H.; .Ramakrishna, S; Huang, Z. M;. Bibo, G.A Comp.: Part A, 31, p197.(2000)

16. Choi, N. S.; Yamaguchi, H. \& Takahashi, K. - J. Comp. Mat., 7, 30 (1996).

17. Joseph, K.;.James, B. e Carvalho, L. H -IPCM-99, International Phenomena in Composite Materials, p.22, Berlin, (1999).

18. Joseph, K. Medeiros, E. S. e Carvalho, L. H. In: Anais do $5^{\circ}$ Congresso Brasileiro de Polímeros, p.693, Águas de Lindóia, São Paulo, (1999)

19. Nóbrega, M. M. S e Carvalho, L. H. in:Anais do SLAP2000, p.236, Havana - Cuba, dez.(2000).

20. http://www.owenscorning.com.br/111a.asp

21. Bayley, J.E. - "Mechanical Properties of Composites Micromechanics". In. Handbook of Polymer-Fiber Composites, cap. 4, F. R. Jones (ed), New York, John Willey \& Sons, Inc. (1994).

22. Mishra, S.; Mohanty, A. K.; Drzal, L. T.; Misra, M.; Parija, S.; Nayak, S. K. \& Tripathy, S. S., Comp. Sci. and Technol., 63, p.1, (2003).

23. Agarwal BD, Broutman LJ. Analysis and Performance of Fiber Composites. New York: John Wiley, 1980.

Enviado: 04/03/05

Reenviado: 05/08/05

Aprovado: 25/08/05 